\title{
THE ROLE OF PROBIOTIC INTERVENTION IN REGULATING GUT MICROBIOTA, SHORT-CHAIN FATTY ACIDS AND DEPRESSION-LIKE BEHAVIOR IN LEAD-EXPOSED RATS
}

\author{
XIAOJUN CHEN ${ }^{1,2}$, SHUJUAN MENG $^{3}$, YONGLI YU ${ }^{3}$, SHUANG LI $^{4}$, LEI WU $^{1}$, and YANSHU ZHANG ${ }^{1,4}$ \\ ${ }^{1}$ North China University of Science and Technology, Tangshan, China \\ School of Public Health \\ ${ }^{2}$ Workers Hospital of Caofeidian District, Tangshan, China \\ Central Laboratory \\ ${ }^{3}$ Tangshan Hongci Hospital, Tangshan, China \\ Department of Pathology \\ ${ }^{4}$ North China University of Science and Technology, Tangshan, China \\ Experimental Animal Center
}

\begin{abstract}
Objectives: The aim of this study was to observe the depression-like behavior changes of rats exposed to lead with or without probiotic intervention, and to investigate changes in the gut microbiota and fecal short-chain fatty acids (SCFAs) levels after lead exposure, and the possible functions of probiotics in this process. Material and Methods: Adult male Sprague Dawley rats were exposed to a $300 \mathrm{mg} / \mathrm{l}$ lead acetate solution for 24 weeks, with or without probiotic (freeze-dried powder containing Lactobacillus and Bifidobacterium: 6 billion live bacteria/2 g) intervention in weeks 17-24. The sucrose preference test (SPT), the forced swim test (FST), and the tail suspension test (TST) were preformed to study the depression-like behaviors of these rats. The alteration of rat gut microbiota induced by lead exposure was analyzed by $16 \mathrm{~S}$ rRNA sequencing, and the levels of fecal SCFAs were detected using gas chromatography. Results: Neurobehavioral tests showed that lead exposure induced depression-like behavior in rats, including reduced sucrose preference in the SPT, and increased immobility times in the FST and the TST. Sequencing and gas chromatography showed that lead exposure changed the structure and the phylogenetic diversity of the gut microbiota, as well as significantly altered the levels of SCFAs. Moreover, the depression-like behaviors, and the changes in both gut microbiota and SCFAs, could be mitigated by probiotic intervention. Conclusions: Lead exposure not only changes the structure and diversity of the gut microbiome but also affects metabolic function. Probiotic intervention may be a novel initiative for the prevention and treatment of neurological damage following lead exposure. Int J Occup Med Environ Health. 2022;35(1):95-106
\end{abstract}

Key words:

depression, lead exposure, probiotic, gut microbiota, SCFAs, neurobehavioral tests

Received: November 21, 2020. Accepted: May 26, 2021.

Corresponding author: Yanshu Zhang, North China University of Science and Technology, School of Public Health, Bohai Road 21, Tangshan, China (e-mail: zhangyanshu70@163.com). 


\section{INTRODUCTION}

Lead is a widespread neurotoxin in production and living environments. Long-term exposure to low concentrations of lead can cause neurological damage, including cognitive impairment, anxiety, depression and other neurological diseases. Depression is mainly characterized by low emotion, low interest, a lack of concentration, slow thinking and behavior, and in severe cases - pessimism, despair and suicidal thoughts. The gut microbiota plays an important role in human health and disease transition, and may also take part in the development of neurological damage caused by lead exposure. With in-depth research on microbial genes and the application of bioinformatics of microbiota, researchers have found that depression is not necessarily a problem of the brain but of gut microbiota; however, the mechanism is still unclear [1-3].

The microbiome-gut-brain axis participates in the bidirectional regulation of the intestinal and central nervous system (CNS) through immune, endocrine, vagus and neurotransmitter pathways [4-6]. Some studies indicate that patients with irritable bowel syndrome often have a psychiatric history, accompanied by gut microbiota disorders. Neuropsychiatric diseases such as autism, depression, and Alzheimer's disease are strongly correlated with the composition of the gut flora [7-9]. The gut flora regulates the secretory reflex and gut movement, as well as the brain and behavior. Bercik et al. [10] found that increases in the abundance of intestinal Citrobacter caused decreases in the number of neurotransmitters in mice, and that the mice presented depressive behavior. Apparently, the gut microbiota shows associations in the junction between the CNS and the gut; therefore, it affects the functional development of the CNS and the occurrence of related diseases.

Short-chain fatty acids (SCFAs) are the end metabolites of dietary fiber fermentation and mainly consist of acetic acid, propanoic acid, butyrate, branched chain amino acids, such as isobutyric acid, and isovaleric acid from protein fermentation, which can affect intestinal mucosal immune balance $[11,12]$. They can have a direct nutritional effect on probiotics, and promote the growth and reproduction of probiotics, but also regulate the $\mathrm{pH}$ value of the gut, prevent the colonization of pathogenic bacteria, promote the proliferation of colon epithelial cells, and maintain intestinal morphology [13]. Short-chain fatty acids are related to intestinal immunity and play an important role in the immunity of the CNS. Researchers have reported that SCFAs produced from a microbial metabolite can reach the circulation and cross the blood-brain barrier to activate specific receptors in the brain regions and neural circuits, associated with depression and anxiety-like behaviors [14-16]. Wu et al. [17] found that the gut flora related to depression affects the production of SCFAs.

Although the potential health risks of lead exposure are widespread, the changes in the intestinal microbiome and SCFAs in the development of lead-induced neurotoxicity are unclear. This study used sequencing and gas chromatography to analyze the composition and relative abundance of gut flora in lead-exposed rats, along with changes in the amounts of fecal SCFAs, and Bifidobacterium and Lactobacillus applied as probiotic interventions in lead-exposed rats, in order to observe the roles of these probiotics in lead-induced depression. The results of this study will provide new strategies for the prevention and treatment of lead-exposed occupational populations.

\section{MATERIAL AND METHODS}

\section{Grouping and treatment of experimental animals}

Forty-five healthy adult male specific pathogen-free Sprague Dawley rats $(200 \pm 10$ g) were obtained and bred in the Experimental Animal Center under the conditions of $20-26^{\circ} \mathrm{C}, 40-70 \%$ relative humidity, and a 12 -hour light and dark cycle, with water provided ad libitum. After a week of adaptive feeding, the animals were randomly divided into 3 groups, i.e., the control group, the leadexposed group, and the intervention group, with 15 animals in each group. The rats in the lead-exposed group 
and the intervention group were exposed to a $300 \mathrm{mg} / \mathrm{l}$ lead acetate solution for 24 weeks. In week 17, probiotics (freeze-dried powder containing Lactobacillus and Bifidobacterium: 6 billion live bacteria/ $2 \mathrm{~g}$, produced by Zhenhe Biological Company of Shanghai, China) were administered to the rats from the intervention group, by gavage, 5 times a week; at least $1.2 \times 10^{9} \mathrm{CFU}$ combined strains were given to each rat, and doses as high as $4.8 \times 10^{10} \mathrm{CFU}$ were administered. The health and activity of the rats were observed daily, and changes in the body weight and water intake were recorded weekly. The animal experimental protocols were reviewed and approved by the Ethics Committee of the North China University of Science and Technology (NCUST, China).

\section{Collection of fecal samples}

After blood collection, the abdominal cavity of the rat was dissected, and the intestinal tissue was separated on a sterilized clean bench. Approximately $300 \mathrm{mg}$ of feces were removed and placed in 2 sterilized $1.5 \mathrm{ml} \mathrm{EP}$ tubes, and rapidly frozen in liquid nitrogen. One of the 2 samples was used for the sequencing of the gut microbiota. The other sample was used to detect the level of SCFAs.

\section{Sucrose preference test (SPT)}

The SPT is a measure of a lack of pleasure, as decreased interest in reward stimulation is a form of neurodepressive behavior. All rats were tested twice. The first test was to determine the preference for basic sugar water, and the second test was to determine the influencing factors. Before the testing experiment, all rats experienced the adaptive phase. A total of $250 \mathrm{ml}$ of a $1 \%$ sucrose solution was placed in the side of the cage and was changed to $250 \mathrm{ml}$ of pure water after $24 \mathrm{~h}$. All rats abstained from drinking for $24 \mathrm{~h}$, which was followed by the beginning of each baseline test. Each rat was given the choice between 2 weighted bottles, one of a $1 \%(\mathrm{w} / \mathrm{v})$ sucrose solution and the other of water. After $2 \mathrm{~h}$, the 2 bottles were weighed and the total liquid consumption was recorded, including both the water consumption and the sucrose consumption of each rat. The following formula was used:

$$
\begin{gathered}
\text { percent sucrose intake }(\%)= \\
\text { sucrose consumption / (sucrose consumption }+ \\
\text { pure water consumption) } \times 100 \% \text {. }
\end{gathered}
$$

\section{Forced swim test (FST)}

The FST is one of the most common experiments to evaluate depression-like behaviors in rodent models. The rats were put into a cylindrical container (diameter: $20 \mathrm{~cm}$, depth: $40 \mathrm{~cm}$ ) with clean water (depth: $25 \mathrm{~cm}$, temperature: $25 \pm 1^{\circ} \mathrm{C}$ ) and forced to swim. Immobility was defined as the absence of all motion with the exception of movements required to keep the head of the rats above water. The duration of immobility in the last $4 \mathrm{~min}$ of a 6-minute test was recorded.

\section{Tail suspension test (TST)}

The rats were individually suspended in a white plexiglass box sized $40 \times 40 \times 40 \mathrm{~cm}, 15 \mathrm{~cm}$ from the ground by the distal end of their tails at $1.5 \mathrm{~cm}$. Immobility was defined as movement without limb struggle, and the total immobility time in the last $4 \mathrm{~min}$ of a 6 -minute test was recorded.

\section{Metagenomic sequencing of the 16S rRNA gene}

Fresh intestinal feces of the rats were quickly collected in a sterilized cryopolymer and stored in a refrigerator at $-80^{\circ} \mathrm{C}$. Total DNA extraction of the microbiome, polymerase chain reaction (PCR) amplification of the target fragment, fluorescence quantification of the amplification product, and high-throughput sequencing of the highly variable V4 region of the $16 \mathrm{~S}$ rRNA gene were then performed. The $\mathrm{V} 4$ region-specific primers were 520F (5'-AYTGGGYDTAAAGNG-3') and 802R (5'-TACNVGGGTATCTAATCC-3'). Then, these obtained se- 
quences were classified by operational taxonomic units (OTUs) with a $97 \%$ threshold of pairwise identity information. The representative sequences of each OTU were used for identification and system analysis. The specific composition of each sample at different taxonomic levels (phylum, class, order, family, and genus) was analyzed. By applying a range of multivariate statistical analysis tools, differences in the bacterial community structure between different samples were further analyzed.

\section{Determination of SCFAs in the feces of rats}

The composition and concentration of SCFAs in the feces of the experimental rats were determined by gas chromatography (GC-MS). First, 50 mg of each fecal sample was accurately weighed and stored; then, $500 \mu \mathrm{l}$ of the solution with a $\mathrm{pH}$ of 1 was added, and the samples were extracted with sufficient vortex oscillation for $10 \mathrm{~min}$. After that, $500 \mu \mathrm{l}$ of ethyl acetate was added, the samples were extracted with vortex oscillation for $30 \mathrm{~min}$, and centrifuged for $10 \mathrm{~min}$ at $12000 \mathrm{r} / \mathrm{min}$, and then the supernatant was collected for measurement. The gas chromatography conditions were as follows: chromatographic column: FFAP elastic quartz capillary column ( $30 \mathrm{~m} \times 320$ microns $\times 1$ microns); heating procedure: the initial temperature was $80^{\circ} \mathrm{C}$, held for $3 \mathrm{~min}$, then it was increased to $120^{\circ} \mathrm{C}$ by increments of $5^{\circ} \mathrm{C} / \mathrm{min}$, and held for $6 \mathrm{~min}$; carrier gas: nitrogen, the flow rate was $20 \mathrm{ml} / \mathrm{min}$; inlet temperature: $200^{\circ} \mathrm{C}$; injection mode: shunting (shunting ratio: 10:1); injection amount: 5.0 units; detector temperature: $240^{\circ} \mathrm{C}$. Data processing was carried out in a gas chromatography workstation at HP Chem Station.

\section{Statistical analysis}

The SPSS software v. 23.0 was used for data statistics and one-way analysis of variance. All the data are expressed as the mean $(\mathrm{M}) \pm$ standard deviation $(\mathrm{SD})$, and $\mathrm{p}<0.05$ indicates a significant difference.

\section{RESULTS}

\section{Effects of body weight and water intake of the experimental rats following lead exposure and probiotic intervention}

The experimental rats were exposed to lead for 24 weeks via drinking water. The health and activity of the rats were observed daily, and their weight and water intake were recorded weekly. As shown in Figure 1a, the weight of the animals in the lead-exposed group was lower than in the control group in weeks 14-24, suggesting that lead exposure caused health problems in the lead-exposed group. Beginning in week 14, the intervention group
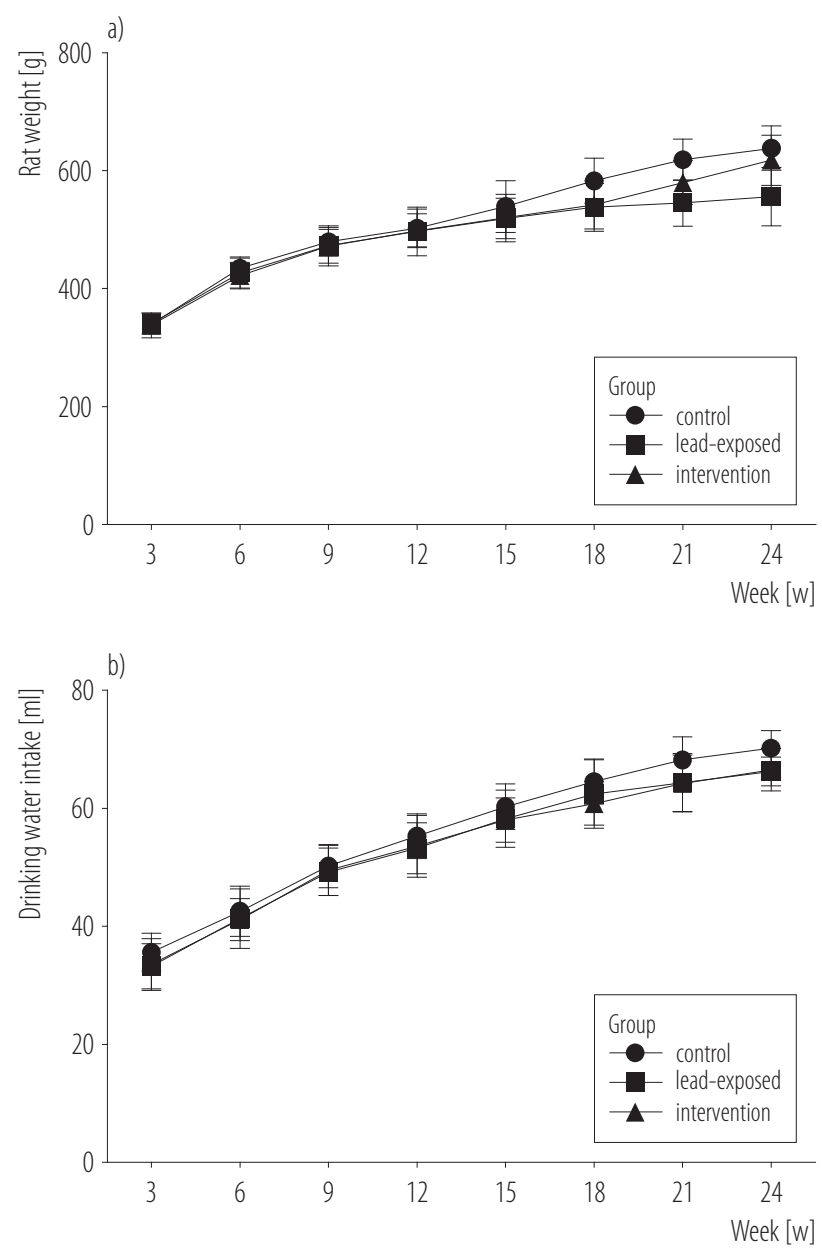

Figure 1. (hanges in a) weight and b) drinking water intake of the rats following lead exposure and Bifidobacterium and Lactobacillus intervention, recorded weekly in September 2017, Tangshan, China 

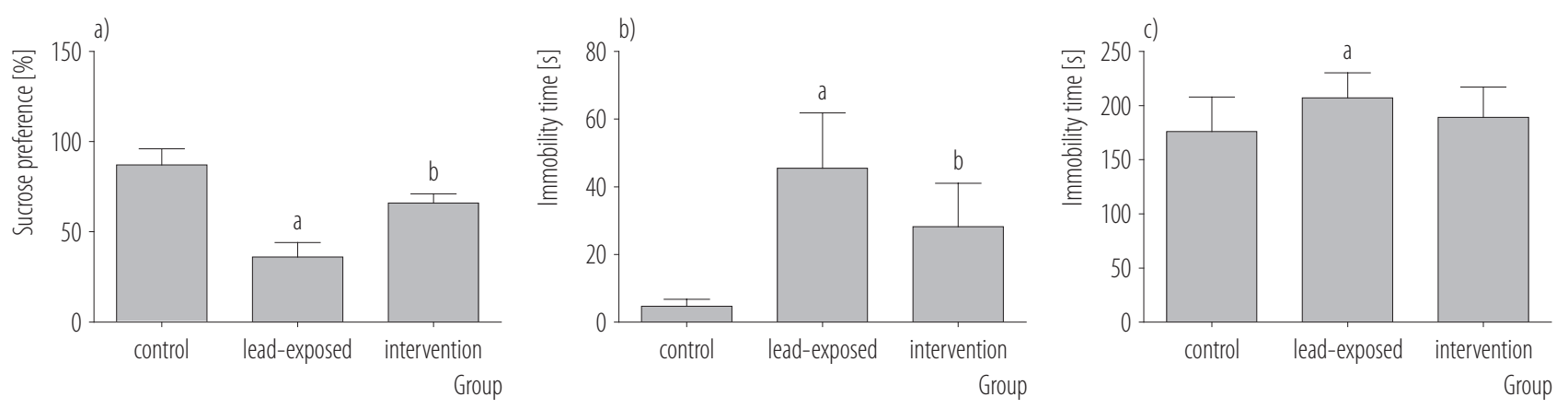

${ }^{\text {a }} \mathrm{p}<0.001$ as compared to the control group.

${ }^{\mathrm{b}} \mathrm{p}<0.001$ as compared to the lead-exposed group $(\mathrm{N}=10)$.

Figure 2. Alteration in depression-like behavior of the rats following lead exposure and Bifidobacterium and Lactobacillus intervention: a) sucrose preference test, b) forced swim test, and c) tail suspension test were used to assess depressive behavior of the rats, September 2017, Tangshan, China

gained more weight compared to the lead-exposed group, and the weight gains were different between the 2 groups. Water intake increased with increasing rat weight, but there was no significant difference between the lead-exposed group and the control group (Figure 1b).

\section{Alterations in the neurobehavior of the experimental rats following lead exposure and probiotic intervention}

The SPT mainly investigates whether the experimental rats lacked pleasure or interest in the stimulation of reward, which is a depression-like behavior. The FST recorded the immobility time within the last $4 \mathrm{~min}$ of a 6-minute test, which was used to evaluate the depressive behaviors of rodent models. The TST was also used to assess the depressive behavior of the rats. In the sugar water preference test, compared to the control group, the sugar water intake of the rats in the lead-exposed group was significantly lower, while that of the intervention group was significantly higher (Figure 2a). In the FST, compared with the control group, the immobility of the lead-exposed group increased, while that of the intervention group experienced a significant decrease (Figure 2b). In the TST, immobility within 2-6 min significantly increased in the lead-exposed group relative to the control group, and that of the intervention group was significantly shortened compared with the lead-exposed group (Figure 2c).

\section{Alteration of $\alpha$-diversity of the gut microbiome in the experimental rats following lead exposure and probiotic intervention}

To analyze the microbes in the feces of the experimental rats, $16 \mathrm{~S}$ rRNA gene sequencing was performed. Among all the samples, the highest number of OTUs was 1888 , and the lowest was 295. As shown in Figure 3a, the OTUs of gut microbiota were compared at the phylum, family and genus levels, and the numbers of OTUs in the leadexposed group were significantly higher than those in the control group and the intervention group. A total of 28098 sequences were randomly selected to statistically analyze the diversity index at a 97\% similarity level of the fecal flora, and the Chaol index and the Shannon index reflected the abundance and evenness, respectively. The Chaol index and the Shannon index were compared between the control group, the lead-exposed group and the intervention group, and the differences between the groups were significant (Figure $3 b$ and $3 c$ ). These data showed that the diversity of the gut microbiota significantly increased in the lead-exposed rats relative to 

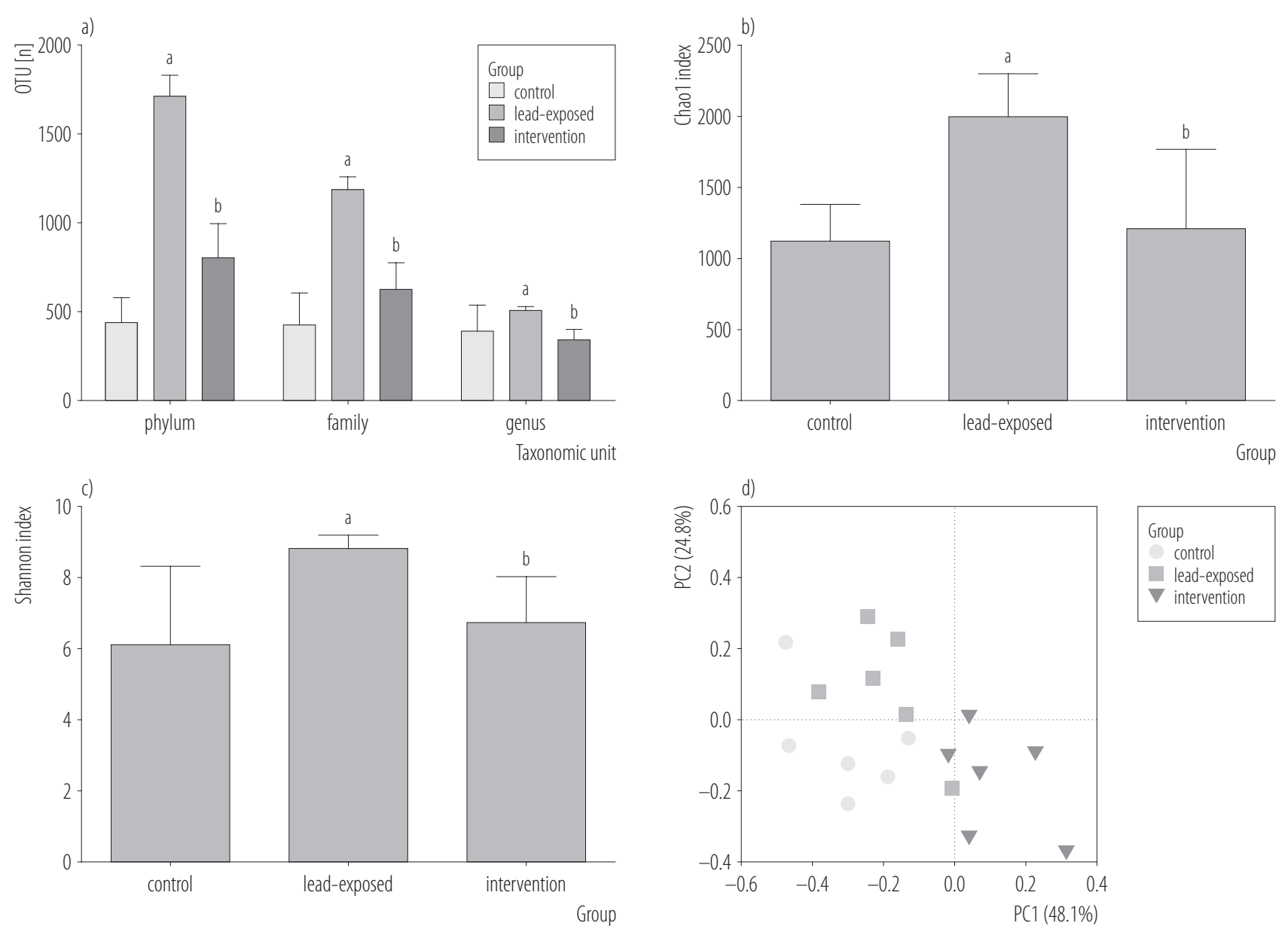

PC1 - the principal coordinate component that can explain the data changes as much as possible; PC2 - the principal coordinate component that accounts for the largest proportion of the remaining changes.

${ }^{\text {a }} \mathrm{p}<0.001$ as compared to the control group.

${ }^{\mathrm{b}} \mathrm{p}<0.001$ as compared to the lead-exposed group $(\mathrm{N}=6)$.

Figure 3. Diversity index of gut microbiota of the rats following lead exposure and Bifidobacterium and Lactobacillus intervention: a) the operational taxonomic units (OTUs) of the intestinal flora in feces from rats, b) the (ha01 index at a 97\% similarity level of the fecal flora data, c) the Shannon index at a $97 \%$ similarity level of the fecal flora data, d) principal coordinates analysis of the similarity of OTUs and the richness of gut flora,

September 2017, Tangshan, China

the control group, while Bifidobacterium combined with Lactobacillus intervention mitigated this effect.

\section{Changes in $\beta$-diversity of the gut microbiota in the experimental rats following lead exposure and probiotic intervention}

To further analyze the difference in the fecal microbial diversity in the experimental rats, OTUs information of each sample was analyzed with a weighted UniFrac distance matrix. Principal coordinates analysis was based on the weighted UniFrac distance matrix and focused on describing the differences in the samples caused by the change in the abundance of community members. As shown in Figure $3 \mathrm{~d}$, the similarity of OTUs and the richness of flora in the control group, the lead-exposed group and the intervention group could be significantly distinguished. 


\section{Effects of the relative abundance}

of the gut microbiome in the experimental rats following lead exposure and probiotic intervention

According to the classification of OTUs, 16S rRNA sequencing was performed on microbial DNA from the feces of the experimental rats, and the composition of the gut microbiota of these rats was determined at the phylum, family and genus levels.

At the phylum level, the microbial community structure and relative abundance of the lead-exposed group and the control group are shown (Figure $4 \mathrm{a}$ and $4 \mathrm{~b}$ ). There was a significant difference between the lead-exposed group and the control group. Compared with the control group, the abundances of Bacteroidetes (15.21\% vs. $6.13 \%$ ) and Proteobacteria (9.59\% vs. $7.60 \%)$ in the lead-exposed group significantly increased, while the Firmicutes abundance decreased $(72.11 \%$ vs. $78.96 \%)$. There were significant differences between the lead-exposed group and the intervention group, and that difference was statistically significant.

At the family level, the microbial community structure and relative abundance of the lead-exposed group and the control group are shown (Figure $4 \mathrm{c}$ and $4 \mathrm{~d}$ ), and there was a significant difference between the lead-exposed group and the control group. Compared with the control group, the abundances of Lactobacillus (2.02\% vs. $27.5 \%)$, Spirochaetes (1.16\% vs. $4.08 \%)$ and Turicibacterales $(0.1 \%$ vs. $3.13 \%)$ in the lead-exposed group significantly decreased. In turn, Ruminococcaceae $(27.47 \%$ vs. $14.49 \%$ ), Clostridium (27.97\% vs. $10.33 \%$ ), and s $24-7$ (10.8\% vs. $4.32 \%)$ were significantly more abundant in the lead-exposed group. The number of Spirobacteria significantly increased in the intervention group.

At the genus level, the microbial community structure and the relative abundances of bacteria in the lead-exposed group and the control group are shown (Figure 4e and $4 \mathrm{f}$ ). There was a significant difference between the lead-exposed group and the control group. Compared with the control group, the abundances of Lactobacillus (2.08\% vs. $29.5 \%)$ and Spirobacteria (1.96\% vs. $4.06 \%)$ in the lead-exposed group significantly decreased. However, Clostridium difficile (69.58\% vs. $46.66 \%$ ) and Bacteroidetes ( $15.21 \%$ vs. $6.12 \%)$ were significantly more abundant. The abundance of the gut flora significantly increased after probiotic intervention.

\section{Changes in fecal SCFAs in the experimental rats following lead exposure and probiotic intervention} The composition and concentration of fecal SCFAs, including acetic acid (Figure 5a), propionic acid (Figure 5b), isobutyric acid (Figure 5c), butyric acid (Figure 5d), valeric acid (Figure $5 \mathrm{e}$ ) and isovaleric acid (Figure $5 \mathrm{f}$ ), were determined through gas chromatography. Compared with the control group, the amounts of fecal acetic acid, propionic acid and butyric acid were significantly decreased in the lead-exposed group, while isobutyric acid, valeric acid and isovaleric acid in each group were not significantly different. Compared with the lead-exposed group, the amounts of fecal acetic acid, propionic acid, butyric acid, isobutyric acid, valeric acid and isovaleric acid in the Bifidobacterium combined with Lactobacillus intervention group were significantly increased.

\section{DISCUSSION}

Depression-like behavior is a common manifestation of emotional disorders, including depression, anhedonia, pessimism, anorexia, severe cognitive impairment, and even suicide [18]. It is known that lead exposure impacts on behavior causing depression, anxiety, and aggressiveness [19]. Although it is generally believed that the richness and diversity of gut microbiota are related to neurological damage, little research has been conducted on the changes in gut microbiota in neurobehavioral disorders following lead exposure.

The present study analyzed the depression-like behaviors, the changes of gut microbiota and fecal SCFAs in lead- 

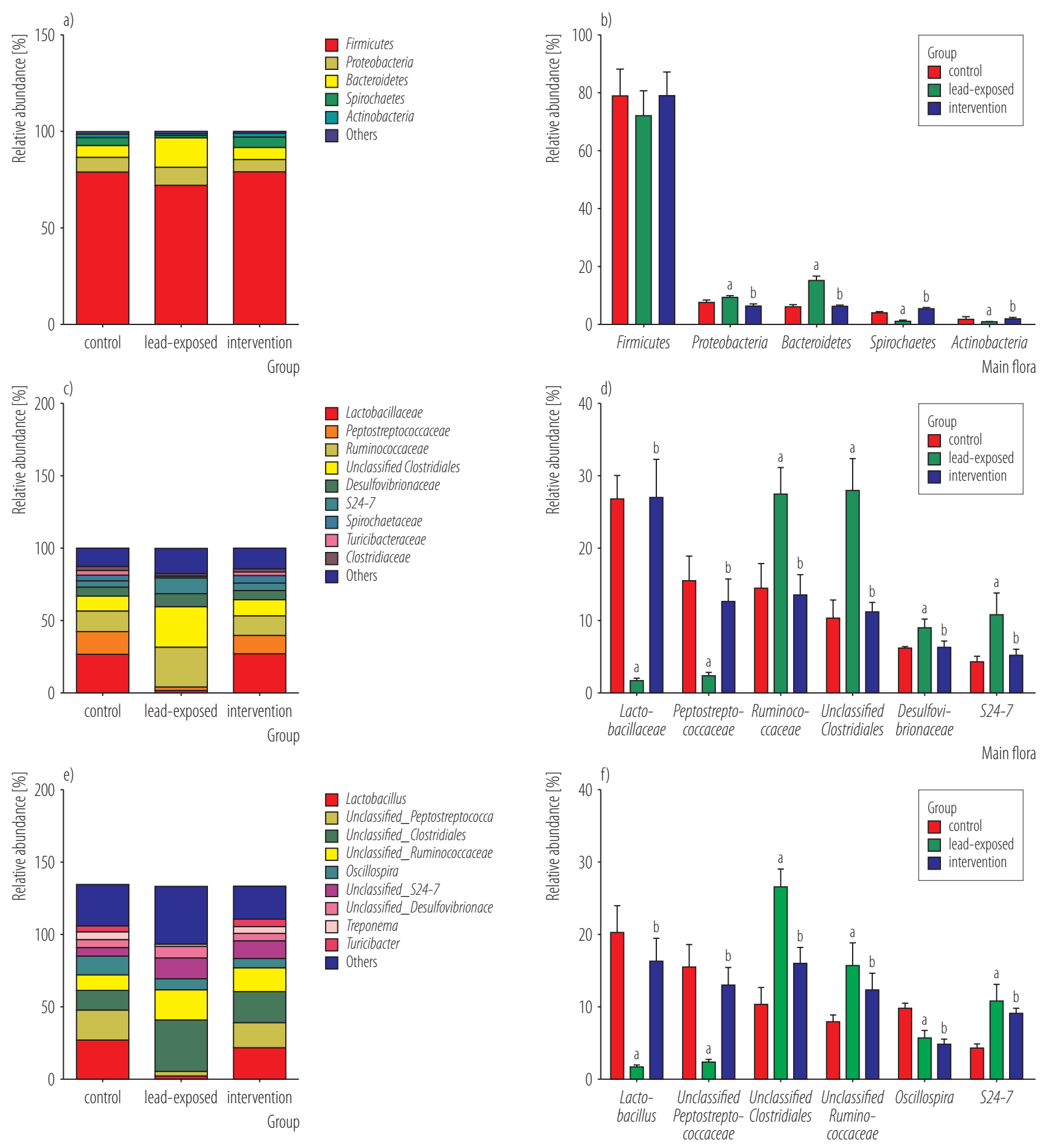

Main flora

${ }^{a} p<0.001$ as compared to the control group.

${ }^{b} \mathrm{p}<0.001$ as compared to the lead-exposed group ( $\left.N=6\right)$.

Figure 4. Relative abundance analysis of gut microbiota - the microbial community structure and relative abundance of the intestinal flora from rat feces: a) and b) phylum level, c) and d) family level, e) and f) genus level, September 2017, Tangshan, China 

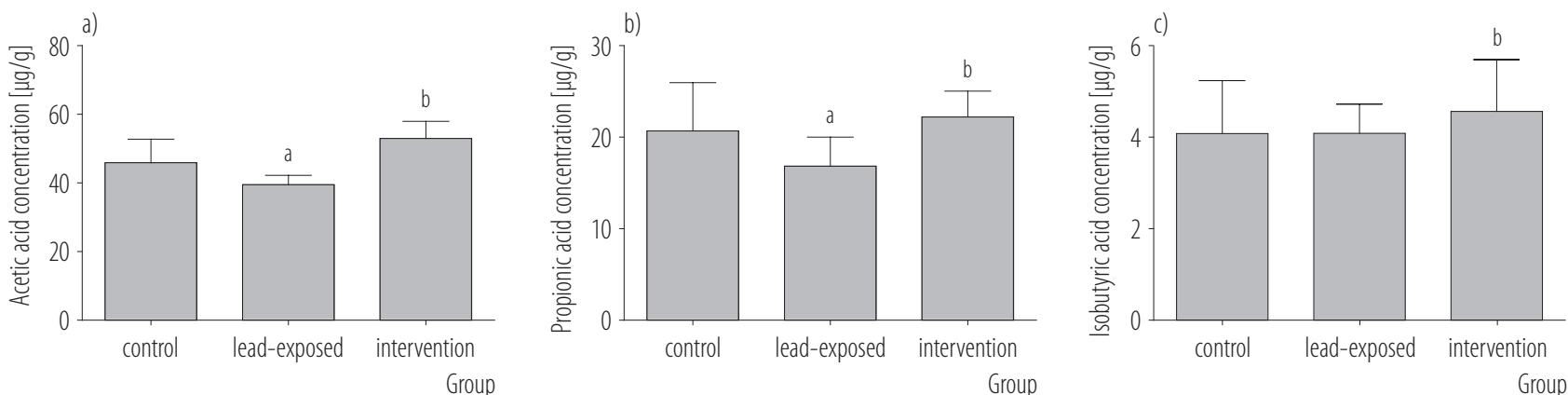

Group
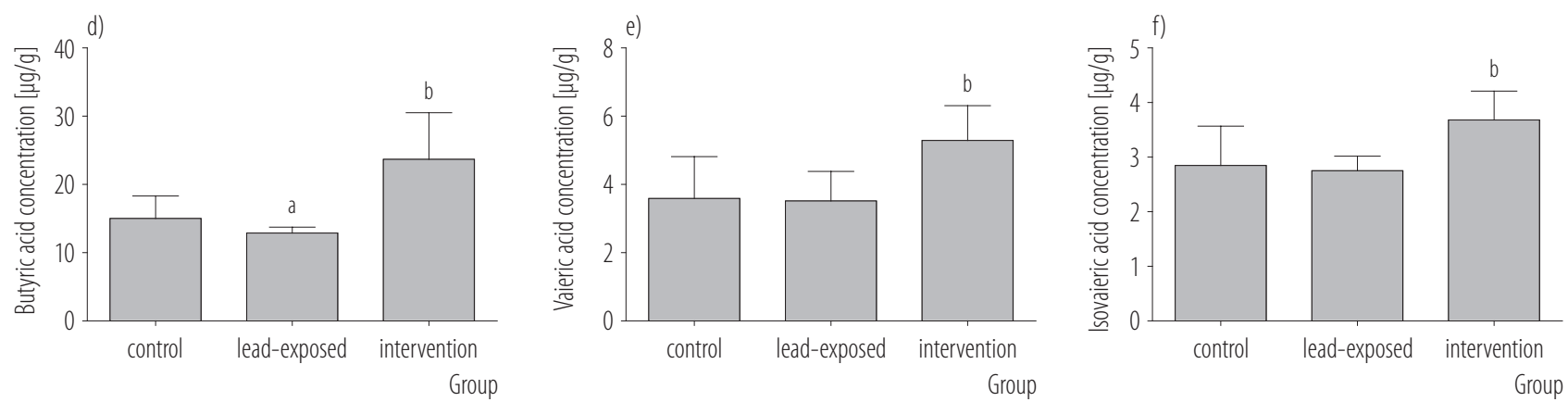

${ }^{a} \mathrm{p}<0.001$ as compared to the control group.

${ }^{\mathrm{b}} \mathrm{p}<0.001$ as compared to the lead-exposed group ( $\left.\mathrm{N}=6\right)$.

The concentrations of fecal SCFAs were determined through gas chromatography.

Figure 5. The short-chain fatty acids (SCFAs) levels: a) acetic acid, b) propionic acid, c) isobutyric acid, d) butyric acid, e) valeric acid, and f) isovaleric acid, in the experimental rats following lead exposure and Bifidobacterium and Lactobacillus intervention, September 2017, Tangshan, China

exposed rats, and the effects of probiotic intervention in this model. Neurobehavior tests showed that lead exposure induced depression-like behavior in rats, accompanying changed structures and the phylogenetic diversity of gut microbiota and altered levels of SCFAs. Moreover, the depression-like behaviors, the changes in gut microbiota and SCFAs could be mitigated by probiotic intervention. The obtained results provided new evidence supporting the regulations between gut microbiota and depression-like behaviors, and new possible mechanism underlying neurotoxicity related to lead exposure. Moreover, this study suggested that probiotic intervention may be used as an adjuvant treatment method for neurobehavioral changes caused by occupations and exposure to environmental factors.

Several studies have proven that gut microbiome regulates the development and function of the brain, and the micro- biome-gut-brain axis works in the interactions between intestinal bacteria and the nervous system through neuroimmune and neuroendocrine pathways [20]. Through the bidirectional communication system, not only the physiological functions of the intestinal tract, including movement, secretion and immune functions, but also the brain functions and behaviors could be affected [21]. Gut microbiota disorders are associated with the occurrence of many neurological diseases, including depression [22,23].

Some studies have reported that mice spontaneously develop depression-like behaviors after fecal transplantation from depressed patients to germ-free (GF) mice, indicating that depression-like behaviors in mice are closely related to the gut microbiome [24]. Xie Peng's team applied cefoxitin, vancomycin and other antibiotics to clear the gut microbiome in mice and to simulate 
GF mice, and found that GF mice exhibited anxiety and depression-like behaviors, and also that this anxiety could be reversed after recolonization of normal flora, while depression-like behaviors could not be reversed [25]. Jiang et al. [26] studied the correlation between depression and the alteration of microbial diversity in human intestinal microflora. They found that there was a significant difference in the composition of intestinal microflora between depressed patients and healthy people, and that the Shannon index was significantly increased in the former group [26]. This finding is consistent with the results presented in this article. Lead exposure in rats induced changes in gut microbiota structures, intestinal microflora diversity alternations, and the increase in the Chao 1 index and the Shannon index.

Besides, the authors found that probiotics, Lactobacillus and Bifidobacterium, showed alleviated effects on the leadinduced depression-like behavior and changes in gut microbiota. It had been reported before that probiotics could stimulate nerve tissue and affect the intestinal and mental health of the host, indicating the effect of probiotics on neuropsychiatric diseases such as depression. The numbers of Lactobacillus and Bifidobacterium significantly decreased in the intestinal tract of depressed people, suggesting their functions in depression, and Lactobacillus and Bifidobacterium supplementation might relieve depression [27]. In rat models where the mother and the infant are separated, treatment with Bifidobacterium can alleviate depression-like behaviors. Feeding Bifidobacterium longum and Lactobacillus helveticus to rats with myocardial infarction, accompanied by depression-like behavior, could reduce the depression-like behavior and the expression of certain inflammatory factors, while also improving the permeability of the intestinal mucosa [28].

Although the authors observed alleviated depression-like behaviors in the Lactobacillus and Bifidobacterium intervention group, and the changes in the gut microbiota structure and diversity, the results showed that the abun- dance of Lactobacillus significantly decreased in the leadexposed group and increased after probiotic intervention, while the probiotic supplement did not increase the abundance of Bifidobacterium. This finding may suggest that Bifidobacteria do not easily colonize the intestines of rats but can promote the rebalancing of gut microbiota. Another important function of the gut microbiome is to produce SCFAs, which affect a series of host processes, including energy utilization, microbial signaling, and $\mathrm{pH}$ changes in the colon, thereby affecting the composition of the gut microbiome and intestinal movement [29]. Studies have reported that Phascolarctobacterium, Roseburia, Blautia, Faecalibacterium, Clostridium, Subdoligranulum, Ruminococcus and Coprococcus in the phylum Firmicutes, as well as bacteria from Bacteroidetes, all have the ability to produce SCFAs by fermentation in the human intestinal tract. Generally, SCFAs are considered to play an important role in maintaining human health [30]. Despite their potentially critical role in the microbiome-gut-brain axis, studies directly linking these acids to neuropsychiatric disorders are relatively rare, and the results are inconsistent or even contradictory.

The results obtained in this study show that Bacteroides, Clostridium and Ruminococcus, which can produce SCFAs, obviously increased in abundance in the leadexposed group, which might be related to the chronic stress of experimental rats following lead exposure. After intervention with Lactobacillus combined with Bifidobacterium, the abundance of Spirobacteria significantly increased, and fecal SCFAs also increased and alleviated the depression-like behavior of the lead-exposed rats.

\section{CONCLUSIONS}

Depression related to lead exposure is associated with changes in both gut microbiota and the levels of fecal SCFAs. Probiotic intervention can relieve the depressionlike behavior of lead-exposed rats and affect the alteration of the gut microbiome, while the fecal SCFAs could be a possible adjuvant therapy of depression. 


\section{ACKNOWLEDGMENTS}

The authors would like to thank all teachers from the School of Public Health and Experimental Animal Center.

\section{REFERENCES}

1. Finegold SM, Downes J, Summanen PH. Microbiology of regressive autism. Anaerobe. 2012;18(2):260-2.

2. Mezzelani A, Landini M, Facchiano F, Raggi ME, Villa L, Molteni M, et al. Environment, dysbiosis, immunity and sexspecific susceptibility: a translational hypothesis for regressive autism pathogenesis. Nutr Neurosci. 2015;18(4):145-61.

3. Ferguson BJ, Marler S, Altstein LL, Lee EB, Mazurek MO, McLaughlin A, et al. Associations between cytokines, endocrine stress response, and gastrointestinal symptoms in autism spectrum disorder. Brain Behav Immun. 2016;58:57-62.

4. Cryan JF, O'Mahony SM. The microbiome-gut-brain axis: from bowel to behavior. Neurogastroenterol Motil. 2011;23(3):187-92.

5. Going JJ, Brewin TB, Crompton GK, McLelland J. Soft tissue sarcoma: two cases of solitary lung metastasis more than 15 years after diagnosis. Clin Radiol. 1986;37(6):579-81.

6. Mayer EA, Tillisch K, Gupta A. Gut/brain axis and the microbiota. J Clin Invest. 2015;125(3):926-38.

7. Gohir W, Whelan FJ, Surette MG, Moore C, Schertzer JD, Sloboda DM. Pregnancy-related changes in the maternal gut microbiota are dependent upon the mother's periconceptional diet. Gut Microbes. 2015;6(5):310-20.

8. Hill-Burns EM, Debelius JW, Morton JT, Wissemann WT, Lewis MR, Wallen ZD, et al. Parkinson's disease and Parkinson's disease medications have distinct signatures of the gut microbiome. Mov Disord. 2017;32(5):739-49.

9. Ghaisas S, Maher J, Kanthasamy A. Gut microbiome in health and disease: Linking the microbiome-gut-brain axis and environmental factors in the pathogenesis of systemic and neurodegenerative diseases. Pharmacol Ther. 2016;158:52-62.

10. Bercik P, Collins SM, Verdu EF. Microbes and the gut-brain axis. Neurogastroenterol Motil. 2012;24(5):405-13.
11. Meng X, Zhou HY, Shen HH, Lufumpa E, Li XM, Guo B, et al. Microbe-metabolite-host axis, two-way action in the pathogenesis and treatment of human autoimmunity. Autoimmun Rev. 2019;18(5):455-75.

12. Parada Venegas D, De la Fuente MK, Landskron G, Gonzalez MJ, Quera R, Dijkstra G, et al. Short Chain Fatty Acids (SCFAs)-Mediated Gut Epithelial and Immune Regulation and Its Relevance for Inflammatory Bowel Diseases. Front Immunol. 2019;10:277.

13. Shinde T, Perera AP, Vemuri R, Gondalia SV, Karpe AV, Beale DJ, et al. Synbiotic Supplementation Containing Whole Plant Sugar Cane Fibre and Probiotic Spores Potentiates Protective Synergistic Effects in Mouse Model of IBD. Nutrients. 2019;11(4).

14. Russo R, Cristiano C, Avagliano C, De Caro C, La Rana G, Raso GM, et al. Gut-brain Axis: Role of Lipids in the Regulation of Inflammation, Pain and CNS Diseases. Curr Med Chem. 2018;25(32):3930-52.

15. Hao Z, Wang W, Guo R, Liu H. Faecalibacterium prausnitzii (ATCC 27766) has preventive and therapeutic effects on chronic unpredictable mild stress-induced depression-like and anxiety-like behavior in rats. Psychoneuroendocrinology. 2019;104:132-42.

16. Stilling RM, van de Wouw M, Clarke G, Stanton C, Dinan TG, Cryan JF. The neuropharmacology of butyrate: The bread and butter of the microbiota-gut-brain axis? Neurochem Int. 2016;99:110-32.

17. Wu M, Tian T, Mao Q, Zou T, Zhou CJ, Xie J, et al. Associations between disordered gut microbiota and changes of neurotransmitters and short-chain fatty acids in depressed mice. Transl Psychiatry. 2020;10(1):350.

18. Otte C, Gold SM, Penninx BW, Pariante CM, Etkin A, Fava M, et al. Major depressive disorder. Nat Rev Dis Primers. 2016;2:16065.

19. Hernandez-Coro A, Sanchez-Hernandez BE, Montes S, Martinez-Lazcano JC, Gonzalez-Guevara E, Perez-Severiano F. Alterations in gene expression due to chronic lead exposure induce behavioral changes. Neurosci Biobehav Rev. 2021;126:361-7. 
20. Wirbisky SE, Weber GJ, Lee JW, Cannon JR, Freeman JL. Novel dose-dependent alterations in excitatory GABA during embryonic development associated with lead $(\mathrm{Pb})$ neurotoxicity. Toxicol Lett. 2014;229(1):1-8.

21. Sylvia KE, Demas GE. A gut feeling: Microbiome-brain-immune interactions modulate social and affective behaviors. Horm Behav. 2018;99:41-9.

22. Sartori SB, Whittle N, Hetzenauer A, Singewald N. Magnesium deficiency induces anxiety and HPA axis dysregulation: modulation by therapeutic drug treatment. Neuropharmacology. 2012;62(1):304-12.

23. Park AJ, Collins J, Blennerhassett PA, Ghia JE, Verdu EF, Bercik $\mathrm{P}$, et al. Altered colonic function and microbiota profile in a mouse model of chronic depression. Neurogastroenterol Motil. 2013;25(9):733-575.

24. Kelly JR, Borre Y, O'Brien C, Patterson E, El Aidy S, Deane J, et al. Transferring the blues: Depression-associated gut microbiota induces neurobehavioural changes in the rat. J Psychiatr Res. 2016;82:109-18.

25. Chen JJ, Xie J, Zeng BH, Li WW, Bai SJ, Zhou C, et al. Absence of gut microbiota affects lipid metabolism in the prefrontal cortex of mice. Neurol Res. 2019;41(12):1104-12.
26. Jiang H, Ling Z, Zhang Y, Mao H, Ma Z, Yin Y, et al. Altered fecal microbiota composition in patients with major depressive disorder. Brain Behav Immun. 2015;48:186-94.

27. Aizawa E, Tsuji H, Asahara T, Takahashi T, Teraishi T, Yoshida S, et al. Possible association of Bifidobacterium and Lactobacillus in the gut microbiota of patients with major depressive disorder. J Affect Disord. 2016;202:254-7.

28. Arseneault-Breard J, Rondeau I, Gilbert K, Girard SA, Tompkins TA, Godbout R, et al. Combination of Lactobacillus helveticus R0052 and Bifidobacterium longum R0175 reduces post-myocardial infarction depression symptoms and restores intestinal permeability in a rat model. Br J Nutr. 2012;107(12):1793-9.

29. Santisteban MM, Qi Y, Zubcevic J, Kim S, Yang T, Shenoy V, et al. Hypertension-Linked Pathophysiological Alterations in the Gut. Circ Res. 2017;120(2):312-23.

30. Bilotta AJ, Cong Y. Gut microbiota metabolite regulation of host defenses at mucosal surfaces: implication in precision medicine. Precis Clin Med. 2019;2(2):110-9.

This work is available in Open Access model and licensed under a Creative Commons Attribution-NonCommercial 3.0 Poland License - http://creativecommons.org/ licenses/by-nc/3.0/pl/deed.en. 